\title{
Comparando a Implantação de Projetos Cooperados baseados no MR-MPS através da Replicação de um Instrumento de Avaliação: Análise Quantitativa sob a ótica das Empresas
}

\author{
Rafael Prikladnicki $^{1,2}$, Odisnei Galarraga ${ }^{1,3}$, Carlos Alberto Becker ${ }^{1,3}$ \\ ${ }^{1}$ SOFTSUL - Agente SOFTEX - POA - RS - Brasil \\ ${ }^{2}$ Faculdade de Informática - PUCRS - POA - RS - Brasil \\ ${ }^{3}$ Software Process Consultoria - POA - RS - Brasil \\ rafaelpepucrs.br, \{odisnei, carlos.becker\} @swprocess.com.br
}

\begin{abstract}
In this paper we present quantitative results of a comparative evaluation conducted with ten companies from the first and second groups of companies implementing the MR MPS. The evaluation considered the companies' point of view and two dimensions: the importance and the satisfaction of some selected criteria. The results are presented and discussed. We believe that the information in this paper can contribute to improve future projects with group of companies in Brazil.
\end{abstract}

Resumo. Neste artigo são apresentados os resultados quantitativos de uma avaliação realizada com dez empresas do primeiro e segundo grupos de um projeto cooperado de implementação do MR MPS. A avaliação considerou a percepção das empresas sob duas dimensões: à importância e a satisfação de dos critérios identificados. Os resultados são apresentados e discutidos. Acredita-se que os dados apresentados neste artigo contribuem para melhorar a execução de futuros projetos cooperados no país.

\section{Introdução}

Nos últimos anos, o Brasil tem vivenciado um crescimento bastante significativo em relação ao investimento na melhoria e qualidade dos produtos de software. Isto tem sido possível, entre outros fatores, pelo crescimento dos programas de melhoria de processo de desenvolvimento de software nas empresas. Diversas empresas vêm implantando melhorias nos seus processos, seguindo modelos de qualidade específicos (Moreira et. al., 2005; Nunes et. al., 2006). Um dos fatores que tem contribuído para este crescimento é o surgimento do programa MPS.BR e seu modelo de referência, o MRMPS (Softex, 2006). Este modelo surgiu em dezembro de 2003, e foi criado a partir da necessidade de padrões de qualidade na indústria de software no Brasil, e que pudesse ser aplicado em empresas com menor poder aquisitivo se comparadas às empresas que investiam em modelos tais como o CMMI (Weber et. al., 2005).

O MPS.BR, segundo Softex (2006) é um programa para melhoria de processo do software brasileiro, e é coordenado pela Associação para Promoção da Excelência do Software Brasileiro (SOFTEX), contando com apoio do MCT, da FINEP e do BID. Ele baseia-se nos conceitos de maturidade e capacidade de processo para a avaliação e 
melhoria da qualidade e produtividade de empresas de software e serviços correlatos. Ele é composto por três componentes: Modelo de Referência (MR-MPS), Método de Avaliação (MA-MPS) e Modelo de Negócio (MN-MPS).

O foco deste artigo é a avaliação comparativa da implantação do Modelo de Negócio Cooperado (MNC-MPS.BR) em dois grupos de cinco empresas cada (duas empresas nível $\mathrm{G}$ e três empresas nível $\mathrm{F}$ em um, e duas empresas nível $\mathrm{F}$ e três empresas nível G no outro), ambos coordenados pela Softsul (agente SOFTEX no RS), em um projeto de implantação do MR-MPS. Os resultados apresentados envolvem a avaliação, por parte das empresas, de um conjunto de itens sob duas dimensões: a importância destes itens para um projeto cooperado, e a satisfação da empresa em relação à execução do projeto.

$\mathrm{Na}$ próxima seção, apresentam-se brevemente os conceitos de um projeto cooperado em grupo de empresas. Na seção 3 é apresentada a metodologia de pesquisa. A seção 4 apresenta os resultados da pesquisa e a seção 5 apresenta as conclusões.

\section{Referencial Teórico}

Projetos em grupos cooperados têm sido bastante utilizados nos últimos anos no Brasil (Wangenheim et al, 2005). Vários grupos cooperados foram organizados, tendo por base o antigo SW-CMM, o CMMI, e agora o MR MPS (Prikladnicki et al, 2007). No MPS.BR, o MNC-MPS.BR prevê a organização de grupos de empresas para a implementação do MR-MPS. Neste modelo, o primeiro passo é a constituição de um grupo de empresas comprometidas com a implementação e avaliação do MR-MPS. Isto pode ocorrer, por exemplo, pela iniciativa de um agente SOFTEX, o que foi o caso deste artigo. Sempre que pertinente, a SOFTEX assina um convênio com a entidade gestora do grupo de empresas (IOGE). A partir de sua constituição, a IOGE assina um contrato com uma das Instituições Implementadoras (II) existentes (MN-MPS.BR, 2007).

O modelo cooperado do MR MPS tem sido uma opção para diversas empresas no país. As empresas buscam alguns benefícios, entre os quais se destacam a divisão dos custos para determinadas atividades, além do compartilhamento de experiências entre as empresas participantes do projeto. Mas não se tem conhecimento, entre os grupos cooperados organizados até então, da execução de avaliações sistemáticas dos projetos, sob a ótica das empresas, conforme o relato apresentado nas próximas seções.

\section{Metodologia de Pesquisa}

Para coletar a opinião das empresas, uma pesquisa do tipo survey (Oates, 2006) foi planejada e aplicada. Esta pesquisa foi aplicada no primeiro grupo, cujos dados foram apresentados em Prikladnicki et al (2007), e replicada no segundo grupo de empresas. O objetivo foi avaliar os itens de maior importância para um projeto cooperado e analisálos frente à satisfação percebida pelas empresas. Além disso, foi feita uma análise comparativa dos dois grupos, de forma a avaliar a evolução da condução de projetos cooperados coordenados pela Softsul, visando também propor um instrumento padrão de avaliação para ser utilizado por outros grupos cooperados em âmbito nacional.

Para a coleta de dados, foi elaborado um questionário com diversos itens a serem avaliados (Anexo 1). Estes itens foram identificados após uma ampla pesquisa bibliográfica, e foram divididos em três 3 grandes grupos: itens relacionados à IOGE, II 
e SOFTEX (I) - nos projetos avaliados, a IOGE e a II eram representadas pela mesma instituição (Softsul) - à empresa (E) e às atividades da cooperativa (C). Todas as questões eram fechadas, em escala Likert (Oates, 2006) variando de 1 (importância ou satisfação mínima) a 5 (importância ou satisfação máxima). Após a elaboração do questionário, o mesmo foi avaliado por três especialistas com conhecimento acadêmico e/ou profissional na área de qualidade de software e no modelo MR-MPS. Além disto, houve a aplicação de um pré-teste envolvendo três profissionais. Para a replicação da avaliação no segundo grupo, o questionário foi revisado por mais dois especialistas, que integram a equipe de implementadores credenciados vinculados a uma II.

Como resultado, houve a revisão do instrumento de pesquisa e a correção de perguntas, de forma a identificar problemas e aumentar o efetivo significado para os respondentes, antes da coleta de dados (Frankfort-Nachmias, 1996). O questionário foi então enviado por e-mail para três representantes de cada empresa (o patrocinador, o coordenador do grupo de melhoria, e um integrante do grupo de melhoria). Ao todo, foram recebidas 24 respostas (11 do primeiro grupo e 13 do segundo grupo). Para a análise dos dados utilizou-se o software SPSS V10.0 (Statistical Package for the Social Sciences). Para efeitos de comparação das avaliações, foi utilizada a estatística descritiva, através do cálculo da média e do desvio padrão dos itens.

\section{Resultados da Pesquisa}

A seguir apresentam-se os resultados comparativos das duas dimensões avaliadas.

\subsection{Importância dos critérios para um projeto cooperado}

Em relação à importância dos itens avaliados para um modelo cooperado, a tabela 1 apresenta os dez mais importantes, na opinião das empresas de cada um dos grupos.

Tabela 1. Itens considerados mais importantes para um projeto cooperado

\begin{tabular}{|c|l|c|c|lc|c|}
\hline \multicolumn{3}{|c|}{ Grupo cooperado 1 } & \multicolumn{3}{c|}{ Grupo cooperado 2 } \\
\hline Dim & Questões & Média & Média & Questões & Dim \\
\hline I & Elaboração do plano de melhoria & 5,00 & 5,00 & Percepção interna das melhorias nos processos & E \\
\hline I & Mentoring presencial & 5,00 & 4,92 & Disponibilidade de recursos pela empresa & E \\
\hline E & $\begin{array}{l}\text { Alinhamento entre processos baseados no MR- } \\
\text { MPS e objetivos e estratégia da empresa }\end{array}$ & 5,00 & 4,92 & Equipe interna de implementação do projeto & E \\
\hline I & Treinamentos realizados & 4,91 & 4,92 & Subsídio financeiro existente & I \\
\hline I & Diagnóstico inicial & 4,91 & 4,85 & Diagnóstico inicial & I \\
\hline E & Equipe interna de implementação do projeto & 4,91 & 4,85 & Elaboração do plano de melhoria & I \\
\hline E & Retorno do investimento & 4,91 & 4,85 & $\begin{array}{l}\text { Alinhamento entre processos baseados no MR } \\
\text { MPS e objetivos e estratégia da empresa }\end{array}$ & E \\
\hline E & Execução de treinamento interno & 4,91 & 4,77 & Mentoring presencial & I \\
\hline I & Elaboração e assinatura do contrato & 4,82 & 4,77 & $\begin{array}{l}\text { Conciliação do trabalho diário com a } \\
\text { implementação dos processos }\end{array}$ & E \\
\hline I & Seminários oferecidos & 4,82 & 4,77 & $\begin{array}{l}\text { Integração entre equipe interna de } \\
\text { implementação e os demais colaboradores }\end{array}$ & E \\
\hline
\end{tabular}

Comparando as médias relativas à importância dos itens, obtidas nas duas pesquisas, observa-se que cinco itens que estavam entre os dez considerados mais importantes pelo primeiro grupo de empresas também estão entre os dez mais importantes para o segundo grupo pesquisado, quais sejam:

- Equipe interna de implementação do projeto;

- Elaboração do plano de melhoria;

- Alinhamento entre processos baseados no MR MPS e objetivos da empresa; 
- Diagnóstico inicial; e

- Mentoring presencial.

É importante para que as instituições responsáveis pelo programa de melhoria (SOFTEX, IOGE e II) adotem ações que permitam melhorar o seu desempenho no que se refere a estas questões. Mesmo nos itens que são de responsabilidade da empresa (E), é interessante que os agentes externos envolvidos no programa cooperado orientem as empresas no sentido de dedicar atenção a estas questões, dada a importância atribuída a elas. Na outra ponta, têm-se os dez itens de menor importância (Tabela 2).

Tabela 2. Itens considerados menos importantes para um projeto cooperado

\begin{tabular}{|c|l|c|c|l|c|}
\hline \multicolumn{2}{|c|}{ Grupo cooperado 1 } & \multicolumn{3}{c|}{ Grupo cooperado 2 } \\
\hline Dim & Questões & Média & Média & Questões & Dim \\
\hline I & Apresentação formal do projeto para empresas & 4,10 & 4,00 & Integração das empresas da cooperativa & C \\
\hline C & Integração das empresas da cooperativa & 4,09 & 4,00 & Workshops em grupo & C \\
\hline I & Equipe administrativa & 4,00 & 4,00 & Seminários em grupo & C \\
\hline C & Apoio ferramental disponibilizado & 4,00 & 3,85 & Mentoring remoto & I \\
\hline I & Equipe de suporte a eventos & 3,91 & 3,62 & Compartilhar expectativas entre as empresas & C \\
\hline I & $\begin{array}{l}\text { Acesso às informações sobre grupos que atuam } \\
\text { no modelo cooperado }\end{array}$ & 3,91 & 3,46 & Equipe administrativa & I \\
\hline C & Similaridade de níveis no grupo cooperado & 3,91 & 3,38 & Equipe de suporte a eventos \\
\hline I & Mentoring remoto & 3,90 & 3,25 & Similaridade na evolução das empresas & I \\
\hline C & Similaridade na evolução das empresas & 3,80 & 3,00 & $\begin{array}{l}\text { Similaridade de níveis em um mesmo grupo } \\
\text { cooperado }\end{array}$ & C \\
\hline C & $\begin{array}{l}\text { Similaridade entre empresas da cooperativa } \\
\text { (objetivos, negócio, etc.) }\end{array}$ & 3,73 & 2,83 & $\begin{array}{l}\text { Similaridade entre empresas da cooperativa } \\
\text { (objetivos, negócio, etc.) }\end{array}$ & C \\
\hline
\end{tabular}

Sete itens aparecem entre os dez menos importantes nos dois grupos:

- Mentoring remoto;

- Equipe administrativa;

- Equipe de suporte a eventos;

- Integração das empresas da cooperativa;

- Similaridade na evolução das empresas;

- Similaridade de níveis em um mesmo grupo cooperado; e

- Similaridade entre empresas da cooperativa (objetivos, negócio, etc.).

O conhecimento destes fatores permite que as mesmas instituições responsáveis pelo programa cooperado destinem maior esforço e recursos aos fatores considerados mais relevantes do ponto de vista das empresas. Por exemplo, o item "mentoring presencial" está entre os dez considerados mais importantes pelas empresas, enquanto que "mentoring remoto" é um dos dez percebidos como menos importantes. Neste caso, em um próximo grupo cooperado, poderia haver um planejamento de mais tempo dos consultores para os mentorings presenciais em relação aos atendimentos remotos.

\subsection{Satisfação dos critérios nas Cooperativas 2006 e 2007 da Softsul}

Em relação à satisfação com os itens avaliados, a tabela 3 apresenta os dez itens que deixaram as empresas mais satisfeitas, em cada um dos grupos cooperados.

Tabela 3. Itens com maior índice de satisfação

\begin{tabular}{|c|l|c|c|l|c|c|}
\hline \multicolumn{3}{|c|}{ Grupo cooperado 1 } & \multicolumn{3}{c|}{ Grupo cooperado 2 } \\
\hline Dim & Questões & Média & Média & Questões & Dim \\
\hline E & $\begin{array}{l}\text { Momento escolhido pela empresa para a } \\
\text { implementação do projeto }\end{array}$ & 4,64 & 4,67 & Execução da avaliação informal & I \\
\hline
\end{tabular}




\begin{tabular}{|c|l|c|c|l|c|}
\hline \multicolumn{3}{|c|}{ Grupo cooperado 1 } & \multicolumn{3}{c|}{ Grupo cooperado 2 } \\
\hline Dim & Questões & Média & Média & Questões & Dim \\
\hline E & Apoio da alta gerência & 4,64 & 4,55 & $\begin{array}{l}\text { Processo de adesão das empresas à } \\
\text { cooperativa }\end{array}$ & I \\
\hline E & Adaptação ao modelo MR-MPS & 4,64 & 4,46 & Apoio da alta gerência & E \\
\hline I & Workshops organizados & 4,55 & 4,45 & $\begin{array}{l}\text { Apresentação formal do projeto para as } \\
\text { empresas }\end{array}$ & I \\
\hline E & Equipe interna de implementação do projeto & 4,55 & 4,42 & Diagnóstico inicial & I \\
\hline I & Equipe técnica & 4,45 & 4,38 & Horas de mentoring presencial & I \\
\hline I & Qualidade do mentoring presencial & 4,45 & 4,25 & $\begin{array}{l}\text { Metas físicas estabelecidas para liberação } \\
\text { dos recursos financeiros da Softex }\end{array}$ & I \\
\hline C & Realização dos seminários em grupo & 4,45 & 4,23 & Coordenação do projeto & I \\
\hline I & Qualidade do mentoring remoto & 4,44 & 4,23 & $\begin{array}{l}\text { Entendimento interno dos benefícios da } \\
\text { implementação }\end{array}$ & E \\
\hline I & Seminários oferecidos & 4,36 & 4,23 & $\begin{array}{l}\text { Alinhamento entre processos do MR-MPS e } \\
\text { objetivos e estratégia da empresa }\end{array}$ & E \\
\hline
\end{tabular}

Se no caso dos níveis de importância foram obtidos resultados semelhantes para os dois grupos pesquisados, o mesmo não ocorreu com os níveis de satisfação. Houve uma diferença considerável entre os resultados obtidos. Dos dez itens com maior índice de satisfação no primeiro grupo, apenas um aparece no segundo grupo:

- Apoio da alta gerência.

Além disso, este item refere-se a questões internas das empresas. Isto significa que alguns itens de responsabilidade das instituições externas às empresas resultaram em uma menor satisfação percebida pelo segundo grupo.

Os demais itens considerados pelo primeiro grupo de empresas entre aqueles com maior índice de satisfação tiveram um nível de satisfação mais baixo na percepção do segundo grupo pesquisado. Isto sugere que, no segundo grupo, várias questões consideradas pelo primeiro grupo como melhor encaminhadas tiveram um desempenho inferior. É importante, neste caso, que sejam investigadas as causas desta menor satisfação do segundo grupo de empresas em relação a estas questões, de forma que elas possam ser objeto de ações corretivas em um novo projeto cooperado.

$\mathrm{Na}$ outra ponta, têm-se os dez itens de menor satisfação (Tabela 4).

Tabela 4. Itens com menor índice de satisfação

\begin{tabular}{|c|l|c|c|l|c|c|}
\hline \multicolumn{4}{|c|}{ Grupo cooperado 1 } & \multicolumn{5}{c|}{ Grupo cooperado 2 } \\
\hline Dim & Questões & Média & Média & Questões & Dim \\
\hline I & Equipe administrativa & 3,67 & 3,60 & Qualidade do mentoring remoto & I \\
\hline I & Processo de adesão de empresas à cooperativa & 3,63 & 3,58 & Compartilhar expectativas entre empresas & C \\
\hline I & Elaboração e assinatura do contrato & 3,63 & 3,54 & Carga horária total do projeto & I \\
\hline C & Similaridade na evolução das empresas & 3,40 & 3,46 & Duração total do projeto & I \\
\hline C & Compartilhar dificuldades entre empresas & 3,36 & 3,46 & $\begin{array}{l}\text { Conciliação do trabalho diário com a } \\
\text { implementação dos processos }\end{array}$ & E \\
\hline C & Compartilhar expectativas entre empresas & 3,20 & 3,46 & Compartilhar soluções entre as empresas & C \\
\hline C & Integração das empresas da cooperativa & 3,20 & 3,38 & Compartilhar dificuldades entre as empresas & C \\
\hline C & Compartilhar soluções entre as empresas & 3,18 & 3,33 & Apoio ferramental disponibilizado & C \\
\hline C & Compartilhar experiências entre empresas & 3,09 & 3,31 & Integração das empresas da cooperativa & C \\
\hline I & $\begin{array}{l}\text { Acesso às informações sobre grupos que atuam } \\
\text { no modelo cooperado }\end{array}$ & 2,33 & 3,18 & Horas de mentoring remoto & I \\
\hline
\end{tabular}

Quatro itens percebidos pelo primeiro grupo como os de menor satisfação permaneceram entre os dez "piores" de acordo com o segundo grupo avaliado:

- Compartilhamento de expectativas entre as empresas;

- Compartilhamento de soluções entre as empresas;

- Compartilhamento de dificuldades entre as empresas; e 
- Integração das empresas da cooperativa.

Todos os itens possuem em comum a integração e compartilhamento de informações entre empresas. Apesar da organização de eventos em conjunto, as empresas não se mostraram satisfeitas com as oportunidades e qualidade do compartilhamento de informação, o que sugere um esforço da IOGE para estimular esta integração em projetos futuros, ou entender os motivos da resistência das empresas.

\subsection{Relação entre Importância e Satisfação}

Após a análise isolada de cada dimensão da pesquisa, foi feita uma análise cruzada, relacionando as duas dimensões. $\mathrm{O}$ objetivo desta análise foi identificar itens que possuíam altos níveis de importância e baixos níveis de satisfação entre os respondentes. A tabela 5 apresenta os itens onde a relação entre importância e satisfação mostrou-se mais crítica. A coluna I/S indica a razão entre a pontuação de importância e satisfação. A variação desta razão é de 0,2 a 5. Quando esta razão é maior do que 1, isto sinaliza que a questão merece atenção, pois possui nível de importância superior ao de satisfação. Isto é particularmente crítico para as questões cujo índice de importância é percebido como alto (média de importância 5 ou próxima deste valor).

\section{Tabela 5. Relação entre importância e satisfação}

\begin{tabular}{|c|l|c|c|c|l|}
\hline \multicolumn{3}{|c|}{ Grupo cooperado 1 } & \multicolumn{3}{c|}{ Grupo cooperado 2 } \\
\hline Dim & Questões & I/S & I/S & Questões & Dim \\
\hline I & $\begin{array}{l}\text { Acesso às informações sobre grupos que já trabalharam ou } \\
\text { trabalham no modelo cooperado }\end{array}$ & 1,68 & 1,38 & $\begin{array}{l}\text { Conciliação do trabalho diário com a } \\
\text { implementação dos processos }\end{array}$ \\
\hline C & Compartilhamento de experiências entre as empresas & 1,38 & 1,36 & Recursos disponibilizados pela empresa & E \\
\hline I & Elaboração do plano de melhoria & 1,35 & 1,27 & Compartilhar soluções entre as empresas & C \\
\hline C & Compartilhamento de soluções entre as empresas & 1,34 & 1,26 & Subsídio Financeiro & I \\
\hline I & Elaboração e assinatura do contrato & 1,33 & 1,26 & Retorno do investimento \\
\hline C & Compartilhamento de expectativas entre as empresas & 1,31 & 1,25 & Apoio ferramental disponibilizado \\
\hline C & Integração das empresas da cooperativa & 1,28 & 1,24 & Elaboração do plano de melhoria \\
\hline C & Compartilhamento de dificuldades entre as empresas & 1,27 & 1,23 & Percepção por parte dos clientes & C \\
\hline I & $\begin{array}{l}\text { Metas físicas estabelecidas para liberação dos recursos } \\
\text { financeiros da Softex }\end{array}$ & 1,27 & 1,23 & $\begin{array}{l}\text { Compartilhamento de dificuldades entre as } \\
\text { empresas }\end{array}$ & I \\
\hline I & Diagnóstico inicial & 1,26 & 1,23 & Percepção interna das melhorias \\
\hline
\end{tabular}

As análises isoladas de importância e de satisfação são importantes para entender a percepção das empresas em relação a esses fatores. Entretanto, para melhorar a condução de programas com grupos de empresas, é fundamental saber se o que é considerado importante pelas empresas está sendo atendido de forma satisfatória. Caso sejam identificados fatores que apresentem uma razão muito alta entre percepção de importância e nível de satisfação das empresas, pode-se atuar sobre esses fatores de forma a dar-lhes mais atenção em programas futuros.

Ao comparar-se a razão entre os dois índices nos dois grupos pesquisados, podese observar que no primeiro grupo todas as questões que apresentavam uma razão mais elevada entre importância e satisfação estavam sob responsabilidade dos agentes externos (I/C). Já no segundo grupo, estas questões aparentemente foram melhor encaminhadas, e metade das questões estavam sob responsabilidade da própria empresa (E), indicando alguma dificuldade interna na condução do programa. Além disso, a maior razão entre importância e satisfação caiu de 1,68 para 1,38 entre os dois grupos.

Analisando os dados da tabela 5, também é possível concluir, por exemplo, que a existência do subsídio financeiro é considerada muito importante e que, no entanto, as 
empresas provavelmente gostariam que ele fosse maior. Ou ainda, que o Plano de Melhoria, apesar de ser considerado importante, deveria ser melhor elaborado.

\section{Considerações finais}

O relato apresentado neste artigo buscou avaliar, sob a ótica das empresas participantes de dois projetos no modelo cooperado, quais os itens considerados importantes e como os itens foram avaliados após a execução dos projetos. Em relação aos resultados, eles representam apenas dois dos diversos grupos cooperados atualmente em execução no país (Rocha, 2006; Wangenheim et al., 2006), mas inova ao apresentar uma análise quantitativa, bem como a replicação da avaliação, visando comparar e melhorar constantemente projetos desta natureza.

Os resultados do estudo não podem ser generalizados para outros projetos cooperados. Apesar de trazer aspectos relevantes que podem ser aproveitados por outras iniciativas semelhantes, os resultados refletem a percepção e ação sob a ótica das empresas que fazem parte de apenas uma IOGE. Desta forma, uma conseqüência imediata deste estudo foi o planejamento de ações para serem colocadas em prática no terceiro grupo cooperado de empresas no RS, iniciado em 2008.

Em relação a perspectivas futuras, entende-se que esta pesquisa apresenta-se como um instrumento importante para que os agentes responsáveis por programas cooperado possam melhorar a condução de outros projetos desta natureza. Neste sentido, acredita-se ser interessante a replicação desta pesquisa em outros projetos cooperados no país, através de uma iniciativa que poderia ser coordenada pela SOFTEX. Por este motivo, está sendo desenvolvida uma ferramenta para automatizar o processo de coleta e análise dos dados, visando não apenas comparar os grupos cooperados de uma forma mais abrangente, mas também buscar melhorias para o programa MPS.BR como um todo.

\section{Agradecimentos}

Agradecemos à todos os profissionais que participaram do estudo, e às empresas que viabilizaram a execução dos dois grupos cooperados de implementação do MR MPS, coordenados pela Softsul. Este trabalho também foi submetido ao ciclo 2008 do PBQPSoftware (Programa Brasileiro de Qualidade e Produtividade em Software).

\section{Referências Bibliográficas}

MN-MPS.BR (2007) "Modelo de Negócio para Melhoria de Processo de Software", Disponível em www.softex.br.

Moreira, L. S. R., Samrsla, V. C, Mora, M. C., Prikladnicki, R., Bossle, R., Back, R. (2005) "Uma Avaliação das Equipes de Projeto em um Momento Pré-Avaliação Oficial de um Programa de Melhoria de Processo de Software visando o CMMI nível 2", In: IV SBQS, Porto Alegre.

Nunes, E. D., Pinto, R., Rocha, A. R., Santos, G. (2006) "MPS.BR Nível E - Uma Avaliação em Verde e Amarelo", In: V SBQS, Vila Velha.

Oates, B. J. (2006) "Researching Information Systems and Computing”, Sage. 
Prikladnicki, R., Furlanetto, T. M., Becker, C. A. (2007) "Uma Análise Quantitativa da Implantação do MR MPS em Projetos Cooperados: Avaliação sob a ótica das Empresas", In: SIMPROS, São Paulo, Brasil.

Rocha, A. R. (2006) "Relatório Semestral da COPPE/UFRJ - Instituição Implementadora MPS.BR" Disponível em www.softex.br.

Softex (2006) "MPS.BR - Melhoria de Processo do Software Brasileiro - Guia Geral versão 1.1", Sociedade SOFTEX, Maio de 2006. Disponível em www.softex.br.

Wangenheim, G. V. C., Pickler, K., Thiry, M., Zoucas, A (2006). "Uma Abordagem para a Modelagem Colaborativa de Processos de Software em Micro e Pequenas Empresas" In: V SBQS, Vila Velha, Brasil.

Weber, K. C., Araújo, E., Machado, C. A. F., Scalet, D., Salviano, C. F., da Rocha, A. R. C. (2005) "Modelo de Referência e Método de Avaliação para Melhoria de Processo de Software - versão 1.0 (MR-MPS e MA-MPS)", In: IV SBQS, POA.

Frankfort-Nachmias, C.; Nachmias, D. (1996). "Research Methods in the Social Sciences", 5. ed., Nova York: Sr. Martin's Press. 
Anexo 1 - Itens avaliados através do questionário ${ }^{1}$

\begin{tabular}{|c|c|c|c|c|c|c|c|c|}
\hline \multirow{2}{*}{ DIMENSÃO } & \multirow{2}{*}{ CRITÉRIOS } & - & - & I & + & + & $\mathbf{N}$ & \multirow{2}{*}{ I e/ou S } \\
\hline & & 1 & 2 & 3 & 4 & 5 & A & \\
\hline IOGE & Apresentacão formal do projeto em um modelo cooperado & & & & & & & les \\
\hline IOGE & Processo de adesão de empresas & & & & & & & les \\
\hline IOGE & Elaboração e assinatura do contrato & & & & & & & les \\
\hline IOGE & Planejamento do projeto & & & & & & & S \\
\hline IOGE & Duração total do projeto & & & & & & & $S$ \\
\hline IOGE & Carga horária total do projeto & & & & & & & $\mathrm{S}$ \\
\hline IOGE & Seminários & & & & & & & les \\
\hline IOGE & Workshops & & & & & & & les \\
\hline IOGE & Treinamento externo & & & & & & & les \\
\hline IOGE & Diagnóstico inicial & & & & & & & les \\
\hline IOGE & Definição de um plano de melhoria & & & & & & & les \\
\hline IOGE & Mentoring presencial & & & & & & & les \\
\hline IOGE & Mentoring remoto & & & & & & & les \\
\hline IOGE & Qualidade do mentoring presencial & & & & & & & $\mathrm{S}$ \\
\hline IOGE & Qualidade do mentoring remoto & & & & & & & $\mathrm{S}$ \\
\hline IOGE & Execução da avaliação informal & & & & & & & $\mathrm{S}$ \\
\hline IOGE & Reunião de lições aprendidas & & & & & & & les \\
\hline IOGE & Equipe técnica & & & & & & & S \\
\hline IOGE & Reuniões de acompanhamento técnico & & & & & & & $\mathrm{I}$ \\
\hline IOGE & Coordenação para o projeto & & & & & & & IeS \\
\hline IOGE & Equipe administrativa & & & & & & & IeS \\
\hline IOGE & Equipe de suporte a eventos & & & & & & & IeS \\
\hline IOGE & Metas físicas estabelecidas & & & & & & & les \\
\hline IOGE & Subsídio financeiro existente & & & & & & & IeS \\
\hline IGOE & Acesso às informações sobre experiências com modelo cooperado & & & & & & & les \\
\hline EMPRESA & Disponibilidade de recursos pela empresa & & & & & & & les \\
\hline EMPRESA & Equipe interna de implementação & & & & & & & les \\
\hline EMPRESA & Conciliação do trabalho diário com a implementação dos processos & & & & & & & les \\
\hline EMPRESA & Integração entre equipe interna de implementação e colaboradores & & & & & & & les \\
\hline EMPRESA & Alinhamento entre processos MR MPS e estratégia da empresa & & & & & & & les \\
\hline EMPRESA & Retorno do investimento & & & & & & & les \\
\hline EMPRESA & Percepção interna das melhorias nos processos & & & & & & & les \\
\hline EMPRESA & Percepção por parte dos clientes & & & & & & & les \\
\hline EMPRESA & Momento da empresa para a execução do projeto & & & & & & & les \\
\hline EMPRESA & Treinamento interno & & & & & & & les \\
\hline EMPRESA & Entendimento interno dos benefícios da implementacão & & & & & & & $\mathrm{s}$ \\
\hline EMPRESA & Modelo cooperado & & & & & & & $\mathrm{S}$ \\
\hline EMPRESA & Comprometimento interno & & & & & & & $\mathrm{S}$ \\
\hline EMPRESA & \begin{tabular}{|l|} 
Motivação dos colaboradores \\
\end{tabular} & & & & & & & $\mathrm{S}$ \\
\hline EMPRESA & Apoio da alta gerência & & & & & & & $\mathrm{S}$ \\
\hline EMPRESA & Adaptação ao modelo MR-MPS & & & & & & & S \\
\hline EMPRESA & Comunicação interna & & & & & & & S \\
\hline EMPRESA & Processo desenvolvido & & & & & & & $S$ \\
\hline Cooperativa & Compartilhamento de expectativas entre empresas & & & & & & & IeS \\
\hline Cooperativa & Compartilhamento de dificuldades entre empresas & & & & & & & IeS \\
\hline Cooperativa & Compartilhamento de soluções entre empresas & & & & & & & IeS \\
\hline Cooperativa & Compartilhamento de experiências gerais entre empresas & & & & & & & les \\
\hline Cooperativa & Integração entre empresas de um projeto cooperado & & & & & & & les \\
\hline Cooperativa & Similaridade de níveis em um mesmo grupo cooperado & & & & & & & $\mathrm{I}$ \\
\hline Cooperativa & Similaridade entre empresas da cooperativa (objetivos, negócio, etc.) & & & & & & & $\mathrm{I}$ \\
\hline Cooperativa & Similaridade na evolução das empresas & & & & & & & IeS \\
\hline Cooperativa & Workshops em grupo & & & & & & & IeS \\
\hline Cooperativa & Seminários em grupo & & & & & & & Ies \\
\hline Cooperativa & Treinamentos em grupo & & & & & & & IeS \\
\hline
\end{tabular}

${ }^{1} \mathrm{Na}$ última coluna, I indica que o item foi avaliado apenas na sua importância, S apenas na satisfação, e I e $\mathrm{S}$ indica que o item foi avaliado em ambos (e posteriormente comparado). 
